\title{
Zybert Effect
}

Josef Zybert

Potential competing interests: The author(s) declared that no potential competing interests exist.

The term used in Zybert Cosmology to differentiate between the Doppler Effect.

Doppler Effect a change in the wavelength of sound in a medium

Zybert Effect a change in the speed of light ( or $\hat{c}$ ) in a vacuum 